\title{
Underestimation of Cognitive Impairment in Older Inpatients by the Abbreviated Mental Test Score versus the Montreal Cognitive Assessment: Cross-Sectional Observational Study
}

\author{
Alexander Emery ${ }^{a} \quad$ James Wells ${ }^{a} \quad$ Stephen P. Klaus ${ }^{b} \quad$ Melissa Mather ${ }^{b}$ \\ Ana Pessoab $^{b}$ Sarah T. Pendlebury ${ }^{a, b}$ \\ a Departments of Medicine and Geratology John Radcliffe Hospital, Oxford University \\ Hospitals NHS Foundation Trust, Oxford, UK; ${ }^{b}$ Wolfson Centre for Prevention of Stroke and \\ Dementia, Nuffield Department of Clinical Neurosciences, University of Oxford, Oxford, UK
}

\section{Keywords}

Abbreviated Mental Test Score · Montreal Cognitive Assessment · Cognitive screening · Internal medicine $\cdot$ Geriatric medicine

\begin{abstract}
Background/Aims: Cognitive impairment is prevalent in older inpatients but may be unrecognized. Screening to identify cognitive deficits is therefore important to optimize care. The 10-point Abbreviated Mental Test Score (AMTS) is widely used in acute hospital settings but its reliability for mild versus more severe cognitive impairment is unknown. We therefore studied the AMTS versus the 30-point Montreal Cognitive Assessment (MoCA) in older ( $\geq 75$ years) inpatients. Methods: The AMTS and MoCA were administered to consecutive hospitalized patients at $\geq 72 \mathrm{~h}$ after admission in a prospective observational study. MoCA testing time was recorded. Reliability of the AMTS for the reference standard defined as mild (MoCA <26) or moderate/severe (MoCA <18) cognitive impairment was assessed using the area under the receiver-operating curve (AUC). Sensitivity, specificity, positive and negative predictive values of low AMTS (<8) for cognitive impairment were determined. Results: Among 205 patients (mean/SD age $=84.9 / 6.3$ years, $96(46.8 \%)$ male, $74(36.1 \%)$ dementia/delirium), mean/SD AMTS was 7.2/2.3, and mean/SD MoCA was 16.1/6.2 with mean/SD testing time $=17.9 / 7.2 \mathrm{~min}$. 96/205 (46.8\%) had low AMTS whereas 174/185 (94\%) had low MoCA: 74/185 (40.0\%) had mild and 100 ( $54.0 \%$ ) had moderate/severe impairment. Moderate/severe cognitive impairment was more prevalent in the low versus the normal AMTS group: 74/83 (90\%) versus $25 / 102$ $(25 \%, p<0.0001)$. AUC of the AMTS for mild and moderate/severe impairment were $0.86(95 \%$
\end{abstract}


$\mathrm{Cl}=0.80-0.93)$ and $0.88(0.82-0.93)$, respectively. Specificity of AMTS $<8$ for both mild and moderate/severe cognitive impairment was high (100\%, 71.5-100, and 92.7\%, 84.8-97.3) but sensitivity was lower $(44.8 \%, 37.0-52.8$, and $72.8 \%, 62.6-81.6$, respectively). The negative predictive value of AMTS $<8$ was therefore low for mild impairment $(10.9 \%, 5.6-18.7)$ but much higher for moderate/severe impairment (75.2\%, 65.7-83.3). All MoCA subtests discriminated between low and normal AMTS groups (all $p<0.0001$, except $p=0.002$ for repetition) but deficits in delayed recall, verbal fluency and visuo-executive function were prevalent even in the normal AMTS group. Conclusion: The AMTS is highly specific but relatively insensitive for cognitive impairment: a quarter of those with normal AMTS had moderate/severe impairment on the MoCA with widespread deficits. The AMTS cannot therefore be used as a "rule-out" test, and more detailed cognitive assessment will be required in selected patients.

\section{Introduction}

Comorbid cognitive disorders are prevalent in older hospitalized people. In general medical wards, up to half of older patients may have dementia, although many are undiagnosed, and delirium rates reach $40 \%$ in the oldest old [1-3]. Cognitive impairment may also occur in association with acute illness in the absence of a dementia or delirium syndrome and predicts future dementia $[4,5]$. Patients with comorbid cognitive disorder are often poorly mobile with increased risk of falls and pressure sores, and may lack capacity to make decisions [3]. Rates of institutionalization and mortality are high [1,3]. Identification of cognitive impairment is therefore necessary as part of holistic care but is often neglected in the acute setting $[6,7]$. Hospital clinicians tend to be focussed on physical illness and are poor at identifying cognitive impairment based purely on impression [8].

Guidelines therefore recommend routine screening for cognitive impairment in older inpatients, but there is no consensus on which tests are best [9-11]. We have previously shown that the widely used 10-point Abbreviated Mental Test Score (AMTS [12]) is feasible and valid in the acute hospital setting [13]. However, available data suggest that the AMTS has a ceiling effect being insensitive to milder deficits [13]. The 30-point Montreal Cognitive Assessment (MoCA) was designed to detect mild cognitive impairment and reportedly takes $<10 \mathrm{~min}$ to administer with scores $<26$ defined as abnormal [14]. The MoCA has been validated in a number of specific conditions including Alzheimer's disease [15], mild cognitive impairment [14], Parkinson's disease [16] and stroke [17], and has largely supplanted the older Mini Mental State Examination which is no longer free to use [18].

We therefore determined the reliability of the AMTS in identifying mild and moderate/ severe cognitive impairment defined by the more detailed MoCA in an unselected sample of older inpatients with unplanned admission to the general hospital. We also examined the relative performance of the individual MoCA subtest items, the time taken to administer the MoCA and the factors associated with longer administration times.

\section{Methods}

The Oxford University Hospitals Trust provides services for all acute internal medicine patients in a population of approximately 660,000 and runs an unselected medical admission system irrespective of age. In a prospective observational study, consecutive patients staying at least $\geq 72 \mathrm{~h}$ on a medical ward from March to May 2013 and from March to July 2017 were examined. Patients were eligible if aged $\geq 75$ years, able to participate in testing and were not

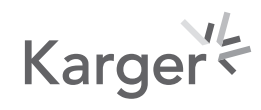


receiving end of life care. Some data on the 2013 cohort have been reported previously [3, 19, $20]$. The study was undertaken to inform future service development as part of a programme to improve the process of care for patients with comorbid cognitive disorders and was approved by the Divisional Management. The study was registered with the Oxford University Hospitals Audit Team (audit registration [datix] No. 2117). Ethics approval was subsequently granted for inclusion in the Oxford Cognitive Comorbidity, Frailty and Ageing Research Database (ORCHARD REC reference 18/SC/0184).

Patients were administered the AMTS [12] followed by the MoCA [14] by A.E., J.W., S.K., M.M., A.P., medical students/clinical fellows trained by S.T.P. Age, sex and educational level were recorded as were any problems that interfered with test administration (e.g., poor vision, inability to use the dominant hand). An additional point for low education ( $\leq 12$ years) was added to the MoCA score in line with the original publication [14]. Diagnoses of dementia and delirium were taken from the clinical record. Time taken to administer the MoCA was calculated by recording the start and end time of MoCA testing with a stopwatch for the 2017 cohort. In addition to the MoCA total and subtest scores, the total number of words produced in $1 \mathrm{~min}$ in the verbal fluency subtest was also recorded. Verbal fluency is a frontal/executive task incorporating elements of processing speed which is not directly measured in the MoCA [21].

Accepted cut-offs on the MoCA of $<26$ were taken to indicate mildly impaired and $<18$ to indicate severely impaired cognitive function $[14,15,17,22]$. The AMTS cut-off of $<8$ was used to define normal versus abnormal AMTS groups [13].

\section{Statistical Analyses}

For calculation of total MoCA scores, patients with any missing subtest item because of problems that interfered with testing were excluded. However, completed subtest items from these patients were included in subtest analyses. Demographic associations (age, sex, education) of MoCA scores were examined using linear regression. The proportion of patients defined as impaired on the AMTS $(<8)$ and with mild $(<26)$ or moderate/severe $(<18)$ impairment on the MoCA were calculated overall. Differences in mean MoCA scores and in categories of none, mild and moderate/severe MoCA-defined impairment between normal and low AMTS groups were compared using ANOVA and $\chi^{2}$ tests, respectively. Similar analyses were undertaken for groups with no dementia/delirium versus dementia/delirium diagnosis.

Reliability of the AMTS for the reference standard of mild and moderate/severe cognitive impairment as defined by the MoCA was assessed using the area under the receiver-operating characteristic curve (AUC). Sensitivity, specificity, positive and negative predictive values for mild and moderate/severe cognitive impairment for different AMTS cut-offs were determined from the AUC.

Mean/SD MoCA subtest scores were determined. Group subtest performance was ranked by calculating the mean subtest score as a percentage of the maximum possible subtest score. Demographic associates (age, sex, education) of MoCA subtest scores were examined using linear regression or logistic regression as appropriate. Comparisons between MoCA subtest scores between those with normal versus abnormal AMTS, and with no dementia/delirium versus dementia/delirium diagnosis, were performed using $t$ tests including the verbal fluency task (number of words produced in $1 \mathrm{~min}$ ).

Associations between total MoCA score and verbal fluency (number of words generated in $60 \mathrm{~s}$ ) and MoCA administration time were determined using linear regression with age, sex, education and problems interfering with testing as covariates. 
Table 1. Study sample overall and by normal versus low AMTS groups and by delirium/dementia diagnosis showing demographic characteristics, rates of mild and moderate/severe cognitive impairment as defined by the MoCA, and MoCA testing time

\begin{tabular}{|c|c|c|c|c|c|c|c|}
\hline & All patients & $\begin{array}{l}\text { Normal } \\
\text { AMTS } \\
n=109\end{array}$ & $\begin{array}{l}\text { Low } \\
\text { AMTS }(<8) \\
n=96\end{array}$ & $p^{\mathrm{a}}$ & $\begin{array}{l}\text { No delirium/ } \\
\text { dementia } \\
n=131\end{array}$ & $\begin{array}{l}\text { Delirium/ } \\
\text { dementia } \\
n=74\end{array}$ & $p^{\mathrm{b}}$ \\
\hline Mean/SD age, years & $84.9 / 6.3$ & $84.6 / 6.5$ & $84.3 / 7.6$ & 0.39 & $83.8 / 7.6$ & $85.9 / 5.8$ & 0.02 \\
\hline Male & $96(46.8)$ & $39(35.8)$ & $57(59.4)$ & 0.84 & $62(44)$ & $34(53)$ & 0.61 \\
\hline Education $<12$ years & $120(58.4)$ & $44(40)$ & $76(79)$ & 0.75 & $83(58.9)$ & $37(57.8)$ & 0.68 \\
\hline MoCA mean $/ S^{c}$ & $16.1 / 6.2$ & $20.1 / 3.9$ & $11.3 / 4.9$ & $<0.0001$ & $17.8 / 5.7$ & $12.9 / 5.8$ & $<0.0001$ \\
\hline MoCA $>26$ & $11(6)$ & $11(11.0)$ & $0(0)$ & $<0.0001$ & $11(9)$ & $0(0)$ & $<0.0001$ \\
\hline MoCA $<26$, >18 & $74(40.0)$ & $66(66.0)$ & $8(10.2)$ & & $56(45.9)$ & $18(28.6)$ & \\
\hline MoCA $<18$ & $100(54.1)$ & $25(25.0)$ & $74(90.1)$ & & $55(45.1)$ & $45(71.4)$ & \\
\hline \multicolumn{8}{|l|}{ Mean/SD MoCA timed, } \\
\hline $\min$ & $17.9 / 7.2$ & $16.4 / 7.7$ & $19.1 / 6.7$ & 0.07 & $17.2 / 7.1$ & $18.9 / 7.7$ & 0.33 \\
\hline
\end{tabular}

${ }^{\mathrm{a}}$ Normal versus low AMTS. ${ }^{\mathrm{b}}$ No delirium/dementia versus delirium/dementia diagnosis. ${ }^{\mathrm{c}} n=185$ completed all subtests on the MoCA. ${ }^{\mathrm{d}} n=91$ had MoCA time taken measured.

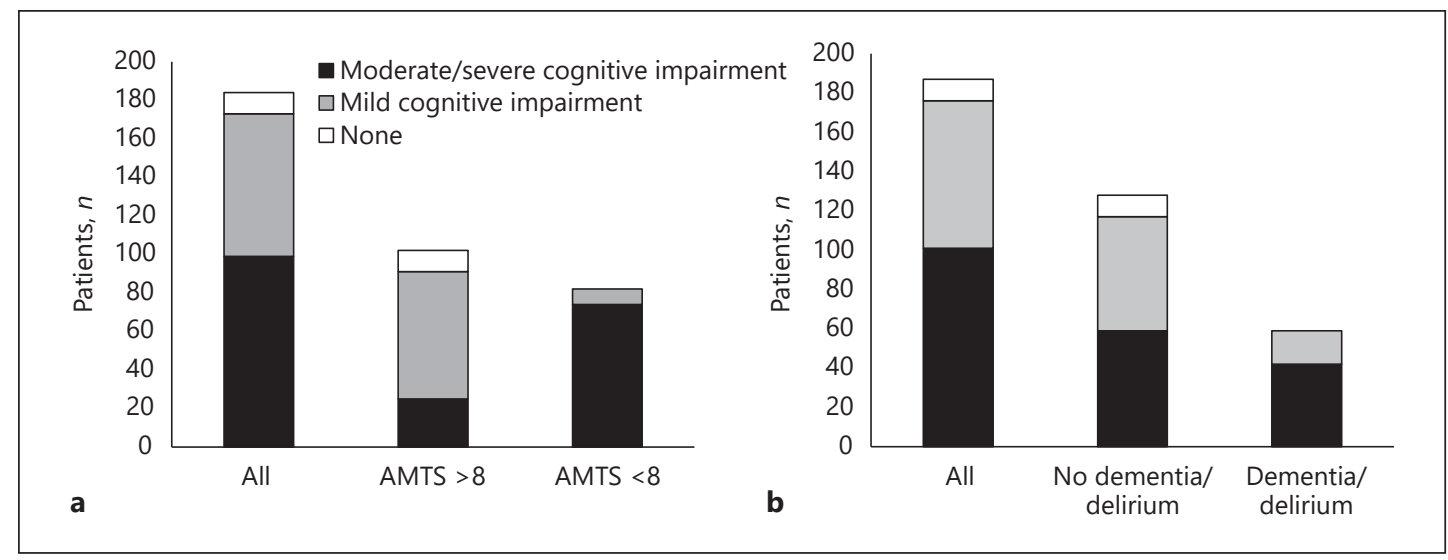

Fig. 1. Number of patients with no cognitive impairment and with mild (MoCA $<26$, light grey shading) and moderate/severe (MoCA $<18$, dark grey shading) impairment in the sample overall, in those with AMTS $\geq 8$ versus AMTS <8 (a) and without diagnosed dementia/delirium versus with dementia/delirium (b).

\section{Results}

Among 205 patients (mean/SD age $=84.9 / 6.3$ years, maximum 102 years, $98(47.8 \%)$ male, $119(58.4 \%)$ education <12 years, $74(36.1 \%)$ dementia/delirium), mean/SD AMTS was 7.2/2.3 (median $(\mathrm{IQR})=8(6,8)$ ) with scores skewed towards normal. Twenty-seven patients had one or more problems that interfered with MoCA testing including poor vision $(n=12)$, deafness $(n=11)$, physical impairment of the upper limb/inability to draw $(n=8)$, dysphasia $(n=1)$, focal discomfort $(n=3)$ and environmental disruption $(n=1)$. For the 185 patients (excluding the 20 patients with poor vision/inability to draw) able to attempt all MoCA subtests including the visuo-executive items, mean/SD MoCA was 16.1/6.2, range $2-28$, and the median (IQR) was $17(11,21)$ with MoCA scores being normally distributed (Table 1). Results were similar after exclusion of all 27 patients with any problem that inter- 
Table 2. Sensitivity, specificity, positive (PPV) and negative predictive values (NPV) for mild and moderate/severe cognitive impairment, as defined by the MoCA, for different cut-offs on the AMTS

\begin{tabular}{|c|c|c|c|c|}
\hline AMTS cut-off & $\begin{array}{l}\text { Sensitivity } \\
(95 \% \text { CI) }\end{array}$ & $\begin{array}{l}\text { Specificity } \\
(95 \% \mathrm{CI})\end{array}$ & $\begin{array}{l}\text { PPV } \\
(95 \% \mathrm{CI})\end{array}$ & $\begin{array}{l}\text { NPV } \\
(95 \% \mathrm{CI})\end{array}$ \\
\hline \multicolumn{5}{|c|}{ Mild cognitive impairment $($ MoCA $<26)$} \\
\hline$<10$ & $83.4 \%(76.8-88.8)$ & $72.7 \%(39.0-94.0)$ & $97.8 \%(93.8-99.6)$ & $22.9 \%(10.4-40.1)$ \\
\hline$<9$ & $61.3 \%(53.4-68.9)$ & $100 \%(71.5-100)$ & $100 \%(96.4-100)$ & $14.9 \%(7.7-25.0)$ \\
\hline$<8$ & $44.8 \%(37.0-52.8)$ & $100 \%(71.5-100)$ & $100 \%(95.1-100)$ & $10.9 \%(5.6-18.7)$ \\
\hline$<7$ & $35.0 \%(27.7-42.8)$ & $100 \%(71.5-100)$ & $100 \%(93.7-100)$ & $9.4 \%(4.8-16.2)$ \\
\hline$<6$ & $20.2 \%(14.4-27.2)$ & $100 \%(71.5-100)$ & $100 \%(89.4-100)$ & $7.8 \%(4.0-13.5)$ \\
\hline \multicolumn{5}{|c|}{ Moderate/severe cognitive impairment $($ MoCA $<18)$} \\
\hline$<10$ & $92.4 \%(84.9-96.9)$ & $34.1 \%(24.0-45.4)$ & $61.2 \%(52.5-69.3)$ & $80.0 \%(63.1-91.6)$ \\
\hline$<9$ & $85.9 \%(77.0-92.3)$ & $74.4 \%$ (63.6-83.4) & $79.0 \%(69.7-86.5)$ & $82.4 \%(71.8-90.3)$ \\
\hline$<8$ & $72.8 \%(62.6-81.6)$ & $92.7 \%(84.8-97.3)$ & $91.8 \%(83.0-96.9)$ & $75.2 \%(65.7-83.3)$ \\
\hline$<7$ & $59.8 \%(49.0-69.9)$ & $97.6 \%$ (91.5-99.7) & $96.5 \%(87.9-99.6)$ & $68.4 \%(59.1-76.7)$ \\
\hline$<6$ & $35.9 \%(26.1-46.5)$ & $100 \%(95.6-100 \%)$ & $100 \%(89.4-100)$ & $58.2 \%(49.6-66.4)$ \\
\hline
\end{tabular}

fered with testing (mean/SD MoCA = 16.4/6.1). Nether MoCA nor AMTS were associated with age, sex or education.

96/205 (46.8\%) had low AMTS, and 174/185 (94\%) testable patients had cognitive impairment on the MoCA overall: $74(40 \%)$ had mild (MoCA $<26, \geq 18)$ and $100(54 \%)$ had moderate/severe impairment $(\mathrm{MoCA}<18)$ with the remaining 11 being unimpaired (Table 1 , Fig. 1). Splitting the patients by low versus normal AMTS showed that MoCA scores were significantly lower in patients with AMTS $<8$ versus $\geq 8$ (mean/SD MoCA $=11.3 / 4.9$ vs. $20.1 / 3.9, p<0.0001$, Table 1$)$. In the low AMTS group, the vast majority $(74 / 82,90 \%)$ had moderate/severe cognitive impairment with the remaining 8 (10\%) having mild impairment, and no patients had a normal MoCA score (Table 1, Fig. 1). In the AMTS $\geq 8$ group, the proportion of patients with moderate/severe impairment was less but it was nevertheless present in 25/102 (25\%), with 66 (65\%) having mild impairment and $11(11 \%)$ being unimpaired (Table 1, Fig. 1). Rates of moderate/severe cognitive impairment were lower in those with a diagnosed cognitive syndrome $(42 / 59,71 \%)$ than in those with low AMTS such that nearly half of those without a diagnosed cognitive syndrome had MoCA $<18$ (55/122, 45.1\%, Table 1, Fig. 1).

AUC of the AMTS for MOCA-defined mild and moderate/severe impairment were 0.86 (95\% CI 0.80-0.93) and 0.88 (0.82-0.93), respectively. The sensitivity, specificity, positive and negative predictive values for mild and moderate severe cognitive impairment by different AMTS cut-offs are shown in Table 2. Specificity of AMTS $<8$ was high for both mild $(100 \%, 95 \% \mathrm{CI}=71.5-100)$ and moderate/severe impairment $(92.7 \%, 84.8-97.3)$. However, sensitivity for mild impairment was low $(44.8 \%, 37.0-52.8)$ whereas it was substantially higher for moderate/severe impairment $(72.8 \%, 62.6-81.6)$. As a result, the negative predictive value for normal AMTS for mild impairment was very low $(10.9 \%, 5.6-18.7)$ but much higher for moderate/severe impairment (75.2\%, 65.7-83.3).

MoCA subtest performance was worst for delayed recall (mean/SD $=0.8 / 1.3,16 \%$ of maximum subtest score), verbal fluency (mean $/ \mathrm{SD}=0.3 / 0.4,30 \%$ of maximum subtest score) and visuo-executive function (mean/SD $=2.3 / 1.5,46 \%$ of maximum subtest score) and best for naming (mean/SD = 2.5/0.8, 83\% of maximum subtest score) (Table 3, Fig. 2).

There were independent associations between demographic variables and some MoCA subtests: age with sentence repetition $(\beta=-0.26, p<0.0001)$ and naming $(\beta=-0.19, p=$ $0.007)$; male sex with calculation $(\beta=0.15, p=0.04)$; and more education with naming $(\beta=$ 
Dementia and

Geriatric Cognitive Disorders Extra
Dement Geriatr Cogn Disord Extra 2020;10:205-215

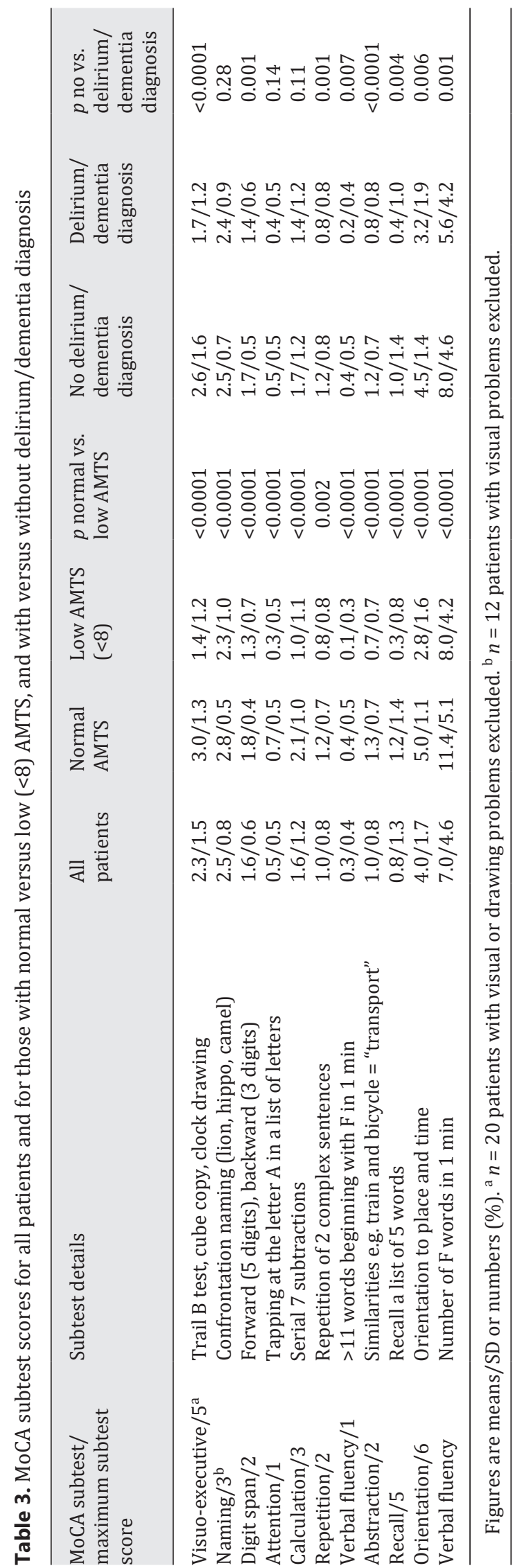




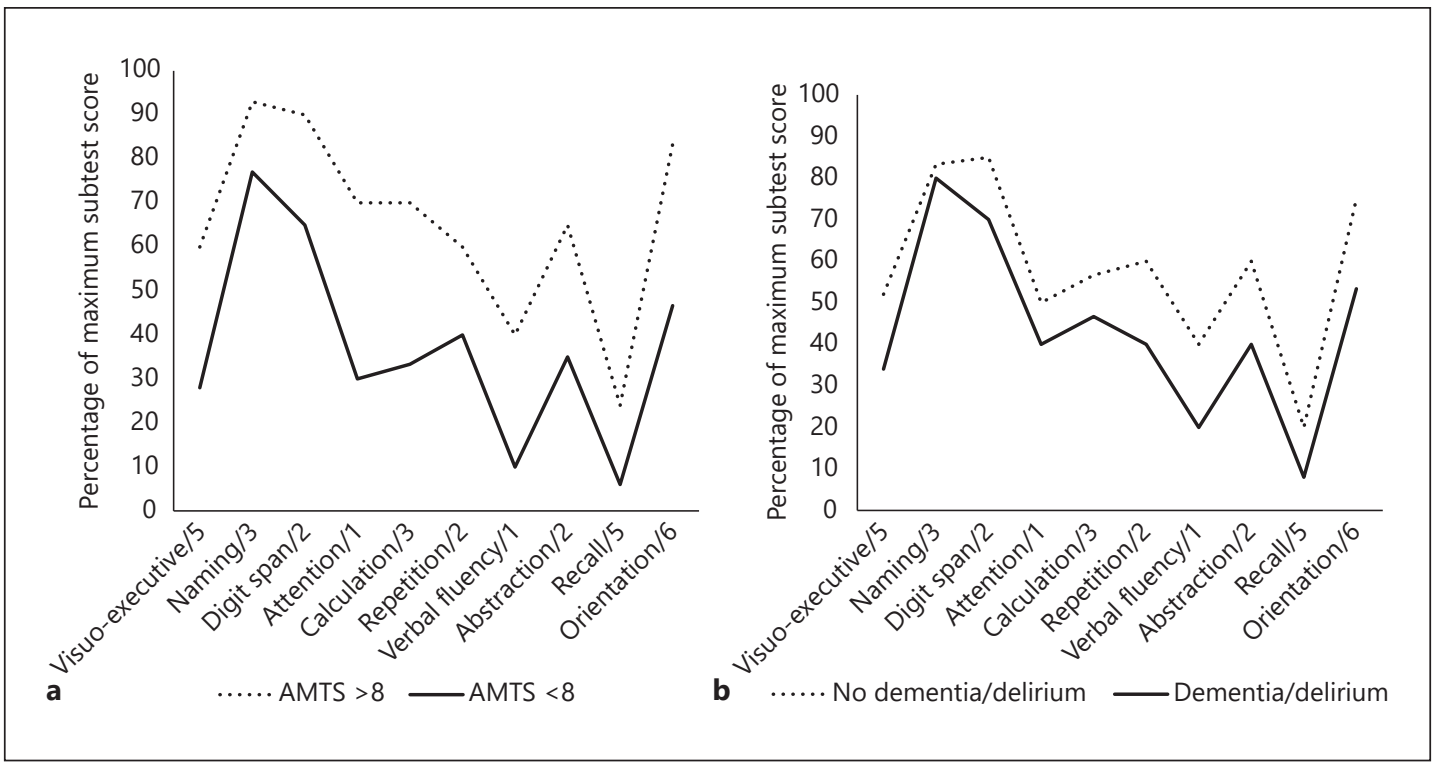

Fig. 2. Mean MoCA subtest scores shown as a percentage of the maximum subtest score. Figures after slash indicate maximum subtest score. a AMTS $\geq 8$ (grey line) and AMTS $<8$ (black line). Differences in subtest performance between groups were significant (all $p<0.0001$, except sentence repetition, $p=0.002$ ). $\mathbf{b}$ Without diagnosed dementia/delirium (grey line) versus with dementia/delirium (black line). Differences in subtest performance between groups were significant except naming, sustained attention and calculation.

$0.14, p=0.04)$ with a trend to calculation $(\beta=0.13, p=0.06)$ and verbal fluency $(\beta=0.13, p=$ $0.07)$. The number of words generated in $60 \mathrm{~s}$ was associated with more education $(\beta=0.14$, $p=0.04$ ).

Comparing normal versus low AMTS groups, showed similar rankings in relative subtest performance but significant differences in the performance of all MoCA subtests with the greatest differences seen for sustained attention (tapping at the letter A), orientation, verbal fluency, visuo-executive function and calculation (Table 3, Fig. 2). However, even in the normal AMTS group, widespread deficits in MoCA subtests were seen (Table 3, Fig. 2). Differences in MoCA subtests in patients with and without a diagnosed dementia/delirium were also seen but were less pronounced than between the normal and abnormal AMTS groups and did not reach significance for naming, sustained attention or calculation (Table 3, Fig. 2).

MoCA time taken was measured in 91 patients (mean/SD age $=83.8 / 8.2$ years, $50(55 \%)$ male, $61(67 \%)$ education $<12$ years). The mean/SD MoCA time taken was 17.9/7.2 min, the median (IQR) was 18 (12-22) min with a range of 3-48 min. In 41/91 (45\%) patients, MoCA took 20 min or more to administer and there was a trend towards longer testing time in patients with low versus normal AMTS (Table 1). MoCA administration time was associated with total MoCA score $(\beta=-0.21, p=0.05)$ and the number of " $F$ " words generated in $60 \mathrm{~s}$ $(\beta=-0.27, p=0.02)$.

\section{Discussion}

Around one half of older inpatients were impaired on the AMTS, but over $90 \%$ were impaired to some degree on the MoCA and moderate/severe cognitive impairment was common. Although sensitivity of the AMTS for mild impairment was low, it was better for 
moderate/severe impairment. MoCA subtest performance showed cognitive deficits across a wide range of cognitive domains including short-term recall, verbal fluency and visuo-executive function even in those with normal AMTS. MoCA administration time was considerably longer than the 10 min stated in the original publication, and nearly half of the patients took at least $20 \mathrm{~min}$.

Our findings have important implications for the interpretation of routine cognitive screening in the general hospital. The AMTS is recommended as a first-line cognitive screen in the acute setting [8-10], and our study shows that AMTS $<8$ is highly specific for cognitive impairment since it invariably indicates the presence of at least mild and in most cases moderate/severe cognitive impairment. However, a "normal" AMTS does not exclude impairment: the negative predictive value for mild impairment was low (only $11 \%$ with normal AMTS were unimpaired on the MoCA). Although the negative predictive value for moderate/severe impairment was higher, a quarter of those with normal AMTS had MoCA < 18 with widespread cognitive deficits. In fact, the older inpatients with normal AMTS performed as poorly on the MoCA as stroke survivors and had a similar cognitive profile with prominent deficits in frontal/executive function as well as recall [17]. This contrasts with the relatively good visuo-executive function seen in Alzheimer's and memory research subjects [23].

Our patients' MoCA scores were broadly comparable (mean MoCA $=16.1$ vs. 19.3) to those reported in internal medicine patients of similar age in a US study designed to compare the MoCA with the Mini Mental State Examination [22]. This study did not report on problems with testing, and MoCA subtests were not examined. We found that problems interfering with testing were common particularly sensory deficits and difficulty drawing as might be expected in older, multi-morbid inpatients. Failure to account for testing problems might result in spurious low scores but are often not reported [24].

Our findings provide information of relevance to the assessment of decision-making capacity in older inpatients. Although capacity assessment is decision and time specific and therefore cannot be inferred from a single cognitive test, the identification of "an impairment of mind or brain" is the first step in any capacity assessment [25]. Staff should be aware of the ceiling effect of the AMTS and that important impairments may be missed using this brief tool (and further, that relying on a diagnosis of dementia/delirium done as part of standard clinical care to identify cognitively impaired patients is even more unreliable $[1,2])$. In addition, the four subsequent components of capacity assessment are the ability to understand, retain, weigh up and communicate a decision [25]. Adequate short-term memory function is required to retain information, and executive function is key to a person's ability to weigh up the risks and benefits of a decision as highlighted in current UK guidance [26]. Tests of executive function are therefore suggested as a useful adjunct to capacity assessment although there is no consensus on which tests to use [26]. The cognitive profile of older inpatients revealed by the MoCA with marked recall and executive deficits would suggest that decision-making capacity is impaired to a greater or lesser extent in the majority of older inpatients including in those with normal AMTS.

All MoCA subtests discriminated between patients with normal versus low AMTS consistent with the larger proportion of severely impaired patients in the low AMTS group. Differences were most pronounced for sustained attention, visuo-executive function and orientation. In contrast, differences in MoCA subtests between patients with and without a clinically diagnosed cognitive syndrome were less apparent probably because of underrecording/diagnosis of these conditions in routine care [1]. The MoCA was designed to be sensitive to mild cognitive impairment in the memory clinic setting, and the relative subtest difficulty has been shown to vary somewhat across disease groups [14-17]. Recall, visuoexecutive function and verbal fluency, along with naming, calculation and orientation, discriminate between controls and mild cognitive impairment subjects [14]. The attentional

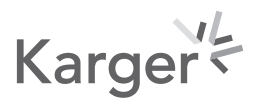


tasks (digit span and tapping at the letter A) show a worse performance in patients with dementia than in normal subjects and those with mild cognitive impairment [14]. We did not see any associations between total MoCA score and age, sex or education in our sample probably because of the restriction to an older age group and the addition of a point for less education as per the original publication [14]. There were associations for some individual subtests including age and naming, sex and calculation, and education and verbal fluency in line with previous studies examining demographic effects on cognitive domain performance $[27,28]$.

The MoCA administration time was considerably longer overall than the $<10$ min stated in the original publication [14]: only a minority of patients were testable in under $10 \mathrm{~min}$, and some took half an hour or longer. Even in those with normal AMTS, the average time taken was over $15 \mathrm{~min}$. There are few data available from other cohorts, but our findings suggest that the resources required, in terms of clinician time, to perform the MoCA in the acute hospital setting are not negligeable. This, together with the problems encountered in testing some patients, make the MoCA unsuitable as a routine screen in this environment. MoCA administration time was inversely associated with total MoCA score, i.e. more impaired patients took longer to perform the test, but problems interfering with testing were not associated possibly because of small numbers. Administration time was also associated with verbal fluency and might therefore be a proxy for processing speed which is not directly measured by the MoCA.

Strengths of our study include the use of a real-world clinical sample of unselected hospitalized older people, the careful recording of problems interfering with testing and the measurement of MoCA administration time, on which there are few data. Weaknesses include the lack of data on rates of untestability in the source sample although previous studies suggest that this is common in older people with severe illness [24, 29]. In addition, the study was undertaken in a single institution. However, this was a large district hospital taking all internal medicine patients from a defined region, and we assessed consecutive unselected patients. The findings are therefore likely to be applicable to other older inpatient populations.

\section{Conclusions}

The AMTS is highly specific for cognitive impairment and is brief and feasible as a routine screen in the acute hospital setting. However, it is poorly sensitive, and a quarter of those with normal AMTS have moderate/severe cognitive impairment with widespread cognitive deficits including executive dysfunction. The AMTS cannot therefore be used to rule out cognitive impairment but more detailed cognitive assessment should be reserved for selected patients where clinically indicated.

\section{Acknowledgements}

S.T.P. is supported by the NIHR Oxford Biomedical Research Centre.

\section{Statement of Ethics}

Written informed consent was not obtained from individual patients in this study. The study was undertaken to inform developments in acute hospital services for frail older patients and was registered with the Oxford University Hospitals Audit Team (audit regis- 
tration [datix] No. 2117). Ethics approval was subsequently granted by the regional research ethics committee (REC reference 18/SC/0184) for use of these data for research purposes as part of the Oxford Cognitive Comorbidity, Frailty and Ageing Research Database (ORCHARD).

\section{Conflict of Interest Statement}

The authors have no conflicts of interest to declare.

\section{Funding Sources}

There was no specific funding for this study.

\section{Author Contributions}

A.E., J.W., S.K., M.M. and A.P. assessed patients and collected data. A.E. and J.W. assisted with data cleaning and assembly. S.T.P. conceived the study as part of an ongoing programme of work to inform the care of older hospitalized patients with cognitive frailty, assembled and cleaned data, performed statistical analyses and wrote the paper.

\section{References}

1 Sampson EL, Blanchard MR, Jones L, Tookman A, King M. Dementia in the acute hospital: prospective cohort study of prevalence and mortality. Br J Psychiatry. 2009 Jul;195(1):61-6.

2 Jackson TA, MacLullich AM, Gladman JR, Lord JM, Sheehan B. Undiagnosed long-term cognitive impairment in acutely hospitalised older medical patients with delirium: a prospective cohort study. Age Ageing. 2016 Jul; 45(4):493-9.

3 Pendlebury ST, Lovett NG, Smith SC, Dutta N, Bendon C, Lloyd-Lavery A, et al. Observational, longitudinal study of delirium in consecutive unselected acute medical admissions: age-specific rates and associated factors, mortality and re-admission. BMJ Open. 2015 Nov;5(11):e007808.

4 Pendlebury ST, Wadling S, Silver LE, Mehta Z, Rothwell PM. Transient cognitive impairment in TIA and minor stroke. Stroke. 2011 Nov;42(11):3116-21.

5 Pendlebury ST, Rothwell PM; Oxford Vascular Study. Incidence and prevalence of dementia associated with transient ischaemic attack and stroke: analysis of the population-based Oxford Vascular Study. Lancet Neurol. 2019 Mar;18(3):248-58.

6 Shakespeare J. An unsafe ward. BMJ. 2013 Feb;346:f1243.

7 Bellelli G, Morandi A, Zanetti E, Bozzini M, Lucchi E, Terrasi M, et al.; AIP delirium study group. Recognition and management of delirium among doctors, nurses, physiotherapists, and psychologists: an Italian survey. Int Psychogeriatr. 2014 Dec;26(12):2093-102.

8 Burleigh E, Reeves I, McAlpine C, Davie J. Can doctors predict patients' abbreviated mental test scores. Age Ageing. 2002 Jul;31(4):303-6.

9 www.england.nhs.uk/wp-content/uploads/2013/02/cquin-guidance.pdf

10 https://www.nice.org.uk/guidance/cmg46/chapter/33-quality-measures\#332-the-best-practice-tariff

11 Jackson TA, Naqvi SH, Sheehan B. Screening for dementia in general hospital inpatients: a systematic review and meta-analysis of available instruments. Age Ageing. 2013 Nov;42(6):689-95.

12 Hodkinson HM. Evaluation of a mental test score for assessment of mental impairment in the elderly. Age Ageing. 1972 Nov;1(4):233-8.

13 Pendlebury ST, Klaus SP, Mather M, de Brito M, Wharton RM. Routine cognitive screening in older patients admitted to acute medicine: abbreviated mental test score (AMTS) and subjective memory complaint versus Montreal Cognitive Assessment and IQCODE. Age Ageing. 2015 Nov;44(6):1000-5.

14 Nasreddine ZS, Phillips NA, Bédirian V, Charbonneau S, Whitehead V, Collin I, et al. The Montreal Cognitive Assessment, MoCA: a brief screening tool for mild cognitive impairment. J Am Geriatr Soc. 2005 Apr;53(4): 695-9.

15 Davis DH, Creavin ST, Yip JL, Noel-Storr AH, Brayne C, Cullum S. Montreal Cognitive Assessment for the diagnosis of Alzheimer's disease and other dementias. Cochrane Database Syst Rev. 2015 Oct;10:CD010775. 
16 Gill DJ, Freshman A, Blender JA, Ravina B. The Montreal cognitive assessment as a screening tool for cognitive impairment in Parkinson's disease. Mov Disord. 2008 May;23(7):1043-6.

17 Pendlebury ST, Mariz J, Bull L, Mehta Z, Rothwell PM. MoCA, ACE-R, and MMSE versus the National Institute of Neurological Disorders and Stroke-Canadian Stroke Network Vascular Cognitive Impairment Harmonization Standards Neuropsychological Battery after TIA and stroke. Stroke. 2012 Feb;43(2):464-9.

18 Folstein MF, Folstein SE, McHugh PR. "Mini-mental state". A practical method for grading the cognitive state of patients for the clinician. J Psychiatr Res. 1975 Nov;12(3):189-98.

19 Pendlebury ST, Lovett N, Smith SC, Cornish E, Mehta Z, Rothwell PM. Delirium risk stratification in consecutive unselected admissions to acute medicine: validation of externally derived risk scores. Age Ageing. 2016 Jan; 45(1):60-5.

20 Pendlebury ST, Lovett NG, Smith SC, Wharton R, Rothwell PM. Delirium risk stratification in consecutive unselected admissions to acute medicine: validation of a susceptibility score based on factors identified externally in pooled data for use at entry to the acute care pathway. Age Ageing. 2017 Mar;46(2):226-31.

21 McDowd J, Hoffman L, Rozek E, Lyons KE, Pahwa R, Burns J, et al. Understanding verbal fluency in healthy aging, Alzheimer's disease, and Parkinson's disease. Neuropsychology. 2011 Mar;25(2):210-25.

22 Saczynski JS, Inouye SK, Guess J, Jones RN, Fong TG, Nemeth E, et al. The Montreal Cognitive Assessment: Creating a Crosswalk with the Mini-Mental State Examination. J Am Geriatr Soc. 2015 Nov;63(11):2370-4.

23 Pendlebury ST, Markwick A, de Jager CA, Zamboni G, Wilcock GK, Rothwell PM. Differences in cognitive profile between TIA, stroke and elderly memory research subjects: a comparison of the MMSE and MoCA. Cerebrovasc Dis. 2012;34(1):48-54.

24 Pendlebury ST, Klaus SP, Thomson RJ, Mehta Z, Wharton RM, Rothwell PM; Oxford Vascular Study. Methodological Factors in Determining Risk of Dementia After Transient Ischemic Attack and Stroke: (III) Applicability of Cognitive Tests. Stroke. 2015 Nov;46(11):3067-73.

25 https://www.nhs.uk/conditions/social-care-and-support-guide/making-decisions-for-someone-else/ mental-capacity-act/

26 https://www.nice.org.uk/guidance/ng108/chapter/

27 O'Connor DW, Pollitt PA, Treasure FP, Brook CP, Reiss BB. The influence of education, social class and sex on Mini-Mental State scores. Psychol Med. 1989 Aug;19(3):771-6.

28 Tombaugh TN, Kozak J, Rees L. Normative data stratified by age and education for two measures of verbal fluency: FAS and animal naming. Arch Clin Neuropsychol. 1999 Feb;14(2):167-77.

29 Bellelli G, Morandi A, Davis DH, Mazzola P, Turco R, Gentile S, et al. Validation of the 4AT, a new instrument for rapid delirium screening: a study in 234 hospitalised older people. Age Ageing. 2014 Jul;43(4):496-502. 